\title{
The Distant Graph of the Ring of Integers and Its Representations in the Modular Group
}

\author{
Andrzej Matraś and Artur Siemaszko@
}

\begin{abstract}
There is presented an infinite class of subgroups of the modular group $\operatorname{PSL}(2, \mathbb{Z})$ that serve as Cayley representations of the distant graph of the projective line of integers. They are infinite countable free products of subgroups of $\operatorname{PSL}(2, \mathbb{Z})$ isomorphic with $\mathbb{Z}_{2}, \mathbb{Z}_{3}$ and $\mathbb{Z}$ subject to the restriction that the number of copies of $\mathbb{Z}$ is 0 or 2 . The proof technique is based on a $1-1$ correspondence between some involutions $\iota$ of $\mathbb{Z}$ that fulfill the equation

$$
\iota\left(\iota(n)-\delta_{n}\right)=\iota(n+1)+\delta_{n+1}, \quad \delta_{n}= \pm 1, \quad \delta_{\iota(n)}=\delta_{n},
$$
\end{abstract}

and groups from this class.

Mathematics Subject Classification. Primary 05C25; Secondary 51C05.

Keywords. subgroups of the modular group, free product of groups, Cayley graph, distant graph, projective line over ring.

\section{Introduction}

Among many constructions of graphs corresponding to a ring in the case of integers the distant graph on the projective line $\mathbb{P}(\mathbb{Z})$ seems most suitable. It is also called the distant graph of the ring of integers. The properties of distant graphs have been studied in many papers, for instance $[1-3,5,6]$. According to the general definition of the distant graph (see i.e. [3]) the vertices are all cyclic submodules of the $\mathbb{Z}$-modul $\mathbb{Z}^{2}$ generated by vectors with co-prime coordinates. The edges of the graph connect vertices whose generators are the rows of an invertible $(2 \times 2)$-matrix over $\mathbb{Z}$. The graph is connected, has three elements maximal cliques and the group $\operatorname{PGL}(2, \mathbb{Z})$ as the automorphism 
group (we omit the proof of the latter fact since it is not crucial for our consideration).

In the paper [6] it was shown that the distant graph on $\mathbb{P}(\mathbb{Z})$ is a Cayley one. There where indicated its two non-isomorphic Cayley groups being subgroups of the modular group and presented a connections with Stern-Brocot tree. The question arises to describe all Cayley groups of this graph. Since the Cayley group of the graph is a subgroup of the automorphism group of this graph, these groups are subgroups of $\operatorname{PGL}(2, \mathbb{Z})$. The paper is a continuation of the last section of [6]. We prove that to any Cayley group of the distant graph on $\mathbb{P}(\mathbb{Z})$ an involution $\iota$ of $\mathbb{Z}$ corresponds satisfying the equation

$$
\iota\left(\iota(n)-\operatorname{det} S_{n}\right)=\iota(n+1)+\operatorname{det} S_{n+1},
$$

with

$$
S_{n}= \pm\left(\begin{array}{cc}
n & -n \iota(n)-\operatorname{det} S_{n} \\
1 & -\iota(n)
\end{array}\right)
$$

and give a recursive construction of a wide class of such involutions. Since we do not know any general method to construct such involutions, we restrict our attention to groups contained entirely in $\operatorname{PSL}(2, \mathbb{Z})$. The latter group we treat as the free product of groups $\operatorname{PSL}(2, \mathbb{Z}) \simeq \mathbb{Z}_{3} * \mathbb{Z}_{2}$ with the presentation

$$
\left\langle T, J: T^{3}=J^{2}=I\right\rangle,
$$

where $T= \pm\left(\begin{array}{cc}0 & -1 \\ 1 & 1\end{array}\right), J= \pm\left(\begin{array}{cc}0 & -1 \\ 1 & 0\end{array}\right)$ and $I= \pm\left(\begin{array}{ll}1 & 0 \\ 0 & 1\end{array}\right)$. Note that $S_{n}$ can be written as

$$
S_{n}=(J T)^{n} J(J T)^{-\iota(n)} .
$$

According to the Kurosh theorem any subgroup of $\operatorname{PSL}(2, \mathbb{Z})$ is a free product of some copies of $\mathbb{Z}_{2}, \mathbb{Z}_{3}$ and $\mathbb{Z}$. We prove that as a Cayley group of the distant graph on $\mathbb{P}(\mathbb{Z})$ we can take any infinite free product of these groups with the restriction that the number of copies of $\mathbb{Z}$ is 0 or 2 .

In the proof we construct an appropriate involution $\iota$ and we use the argumentation of Marshall Hall Jr. from the paper [4] to reveal the form of $\left\langle\left\{S_{n}: n \in \mathbb{Z}\right\}\right\rangle$. In a free product he introduced a so called semi-alphabetical order denoted by $\prec$. In the subgroup $\mathcal{G}$ of a group $\mathcal{H}$ he finds a set $\mathcal{K}=\mathcal{K}(\mathcal{G})$ which consist of all nonidentical elements $K \in \mathcal{G}$ such that $K$ does not belong to the group generated by elements that precede $K$ in the semi-alphabetical order. By this proof the subgroup $\mathcal{G}$ is the free product of groups generated by elements of the set $\mathcal{K}$. To find the set $\mathcal{K}$ for our groups we introduce three properties of subsets of $\operatorname{PSL}(2, \mathbb{Z})$.

We say that the set $\mathcal{F}$ of elements of the modular group has a property

(G) if it contains no product of elements from $\mathcal{F} \cup \mathcal{F}^{-1}$;

$(\mathrm{P})$ if for any $F_{j} \in \mathcal{F}$ and $\varepsilon_{j} \in\{-1,1\}, j=1, \ldots, t, t \geq 2$ with $F_{j}^{\varepsilon_{j}} F_{j+1}^{\varepsilon_{j}+1} \neq I$ one has $\left|F_{1}^{\varepsilon_{1}} \ldots F_{t}^{\varepsilon_{t}}\right|>\left|F_{j}\right|$; 
(I) if $F \preceq F^{-1}$ for each $F \in \mathcal{F}$.

Lemma 1.1. Let $\mathcal{G}$ be a subgroup of the modular group. If some $\mathcal{F} \subset \mathcal{G}$ generates $\mathcal{G}$ and satisfies $(\mathrm{G}),(\mathrm{P})$ and $(\mathrm{I})$ then $\mathcal{F}=\mathcal{K}(\mathcal{G})$.

Proof. Assume that $G \in \mathcal{G} \backslash \mathcal{K}$, i.e $G=G_{1} \ldots G_{k}, k \geq 2$, where each $G_{j} \in \mathcal{G}$ precedes $G$. Since $\mathcal{F}$ generates $\mathcal{G}$, each $G_{j}$, hence $G$ as well, is a non-trivial product of elements of $\mathcal{F} \cup \mathcal{F}^{-1}$. We get $G \notin \mathcal{F}$, by the property (G).

On the other hand, if $G \in \mathcal{G} \backslash \mathcal{F}$ then either $G=F^{-1}$ for some $F \in \mathcal{F}$ or $G$ must be a product of some elements of $\mathcal{F} \cup \mathcal{F}^{-1}$. Therefore $G \notin \mathcal{K}$, in the former case by the property (I) and in the latter case by the property $(\mathrm{P})$.

\section{Involutions of $\mathbb{Z}$}

Assume now that the distant graph on $\mathbb{P}(\mathbb{Z})$ has a Cayley group $(\mathcal{G}, \mathcal{S})$. Since our graph is vertex-transitive, we may assume that the neutral element of $\mathcal{G}$ corresponds to $e= \pm\left[\begin{array}{l}1 \\ 0\end{array}\right]$. Its neighbourhood consists of $v_{n}= \pm\left[\begin{array}{c}n \\ 1\end{array}\right] \in \mathbb{P}(\mathbb{Z})$, $n \in \mathbb{Z}$, hence $\mathcal{S}=\left\{S_{n}: n \in \mathbb{Z}\right\}$, where $S_{n}$ corresponds to $v_{n}$. Two vertices $v_{k}$ and $v_{l}$ are adjacent iff $\left|\operatorname{det}\left[v_{k}, v_{l}\right]\right|=|k-l|=1$. Therefore elements of $\mathcal{S}$ have to satisfy

$$
S_{k}^{-1} S_{l} \in \mathcal{S} \quad \text { iff } \quad|k-l|=1 .
$$

It is not difficult to see that the automorphism group of our distant graph is isomorphic to $\operatorname{PGL}(2, \mathbb{Z})$. This fact allows us to treat elements of $\mathcal{G}$ as members of this group. As $\mathcal{S}=\mathcal{S}^{-1}$ we have $S_{n}^{-1}=S_{\iota(n)}$ for some bijection $\iota: \mathbb{Z} \longrightarrow \mathbb{Z}$. Since $S_{\iota(\iota(n))}=S_{\iota(n)}^{-1}=S_{n}, \iota$ is an involution. We have

$$
S_{n}(e)=v_{n}
$$

If $S_{n}= \pm\left(\begin{array}{ll}a & b \\ c & d\end{array}\right)$, from the above equation applied to $n$ and $\iota(n)$ we get

$$
a= \pm n, \quad c= \pm 1, \quad d=\mp \iota(n) .
$$

Therefore

$$
S_{n}= \pm\left(\begin{array}{cc}
n & -n \iota(n)-\operatorname{det} S_{n} \\
1 & -\iota(n)
\end{array}\right)
$$

are the only candidates for members of $\mathcal{S}$. Straightforward calculations show that (2.1) holds iff $\iota$ satisfies

$$
\iota\left(\iota(n)-\delta_{n}\right)=\iota(n+1)+\delta_{n+1}, \quad \delta_{n}= \pm 1, \quad \delta_{\iota(n)}=\delta_{n},
$$

with $\delta_{n}=\operatorname{det} S_{n}$. In the paper [6] we found two non-isomorphic Cayley groups $\mathcal{G}$ of the distant graph on $\mathbb{P}(\mathbb{Z})$ satisfying the additional condition

$$
(J T)^{n} \in \mathcal{G} \quad \text { iff } \quad n=0 .
$$

In the very end we will use the following observation. 
Lemma 2.1. There is $1-1$ correspondence between involutions $\iota: \mathbb{Z} \longrightarrow \mathbb{Z}$ satisfying (2.2) and the generating sets $\mathcal{S}=\left\{S_{n}: n \in \mathbb{Z}\right\}$ of Cayley group representations $(\mathcal{G}, \mathcal{S})$ of the distant graph on $\mathbb{P}(\mathbb{Z})$.

The proof of the lemma in one direction is above and in the other follows from the considerations after Lemma 4.3 of [6] (at that point we use (2.3)). Therefore, in order to construct a Cayley group of the distant graph on $\mathbb{P}(\mathbb{Z})$ it is enough to find an involution of $\mathbb{Z}$ satisfying (2.2). For this we define some type of involutions on finite sets of consecutive integers. A map $\tilde{\iota}:\{k, \ldots, k+$ $l\} \longrightarrow\{k, \ldots, k+l\}, k \in \mathbb{Z}, l \geq 0$, is called a building involution if:

- $\tilde{\iota}$ is an involution of $\{k, \ldots, k+l\}$;

- $\tilde{\iota}(k)=k+l$;

- $\tilde{\iota}$ satisfies $(2.2)$ for each $n=k, \ldots, k+l-1$.

We can freely shift the domain of a building involution along integers. We now present a recursive method of constructing required involutions.

Lemma 2.2. If $\left(\iota_{n}\right)_{n \geq 0}$ is a sequence of building involutions with domains $D_{n}$ then there exists an involution $\iota$ satisfying (2.2) such that $\left.\iota\right|_{D_{n}}=\iota_{n}$, after the appropriate shift of $D_{n}$.

Proof. Given two building involutions one may construct another one. Indeed, let

$$
\iota_{j}:\left\{k_{j}, \ldots, k_{j}+l_{j}\right\} \longrightarrow\left\{k_{j}, \ldots, k_{j}+l_{j}\right\},
$$

$j=0,1$ be building involutions with $k_{1}=k_{0}+l_{0}+1$. Define

$$
\tilde{\iota}=\iota_{0} \sqcup \iota_{1}:\left\{k_{0}-1, \ldots, k_{1}+l_{1}+1\right\} \longrightarrow\left\{k_{0}-1, \ldots, k_{1}+l_{1}+1\right\}
$$

by

- $\tilde{\iota}\left(k_{0}-1\right)=k_{1}+l_{1}+1$;

- $\left.\tilde{\iota}\right|_{\left\{k_{j}, \ldots, k_{j}+l_{j}\right\}}=\iota_{j}, j=0,1$.

Let us choose a sequence of building involutions $\iota_{n}:\left\{k_{n}, \ldots, k_{n}+l_{n}\right\} \longrightarrow$ $\left\{k_{n}, \ldots, k_{n}+l_{n}\right\}, n \in \mathbb{N}$ satisfying $k_{0}=-1, k_{1}=l_{0}, k_{n+1}=k_{n}+l_{n}+2$ for $n \geq 1$. We define an involution $\iota=\bigsqcup_{n=0}^{\infty} \iota_{n}: \mathbb{Z} \longrightarrow \mathbb{Z}$ as a "limit" construction:

$$
\iota_{0}, \iota_{0} \sqcup \iota_{1}, \iota_{0} \sqcup \iota_{1} \sqcup \iota_{2}, \ldots, \iota_{0} \sqcup \iota_{1} \sqcup \iota_{2} \sqcup \ldots \sqcup \iota_{n}, \ldots
$$

i.e. we require that $\left.\iota\right|_{\left\{-n-1, \ldots, k_{n}+l_{n}+1\right\}}=\iota_{0} \sqcup \iota_{1} \sqcup \iota_{2} \sqcup \ldots \sqcup \iota_{n}$ for each $n \geq 1$. It follows immediately from the construction that $\iota$ thus defined satisfies (2.2).

It is possible to find an involution of $\mathbb{Z}$ satisfying (2.2), with $\delta_{n}=-1$ for some $n \in \mathbb{Z}$.

Example. Let $l_{0}=6$ and $l_{n}=0$ for $n \geq 1$. Define the involution $\iota_{0}$ of the set $\{-1,2,3,4,5\}$ by $\iota_{0}(-1)=5, \iota_{0}(0)=3, \iota_{0}(1)=1, \iota_{0}(2)=4$ and assume that $\delta_{n}=-1$ iff $n \in\{0,2,3,4\}$. It may be checked by inspection that then $\iota_{0}$ satisfies the condition (2.2). Then we use Lemma 2.2 with an arbitrary sequence of building involutions $\left(\iota_{n}\right)_{n=1}^{\infty}$. 
Since we do not know any general method to construct such involutions, we will restrict our attention to groups contained entirely in $\operatorname{PSL}(2, \mathbb{Z})$. With this assumption (2.2) becomes

$$
\iota(\iota(n)-1)=\iota(n+1)+1 .
$$

\section{Constructions of Cayley Representations}

Given $p \in \mathbb{N}$ let us denote by $\mathcal{F}_{p}, \mathcal{T}_{p}$ and $\mathcal{D}_{p}$ the free group with $p$ free generators, the free product of $p$ copies of $\mathbb{Z}_{3}$ and the free product of $p$ copies of $\mathbb{Z}_{2}$, respectively. With such notations we have the following result.

Theorem 3.1. For every three numbers $p, q, r \in \mathbb{N} \cup\left\{\aleph_{0}\right\}$ with $p \in\{0,2\}$ and $p+q+r=\aleph_{0}$ the group

$$
\mathcal{F}_{p} * \mathcal{T}_{q} * \mathcal{D}_{r}
$$

is a Cayley group of the distant graph on $\mathbb{P}(\mathbb{Z})$.

Proof. First we deal with the case $p=0$.

Observe that if $l_{n} \in\{0,1\}$ then $\iota_{n}$ is uniquely determined. Moreover if $l_{n}=0$ then $S_{k_{n}}$ is an involution in $\operatorname{PSL}(2, \mathbb{Z})$, and if $l_{n}=1$ then $S_{k_{n}}$ is of order 3. It follows that in the case we deal with in order to define $\iota$ it is enough to choose a sequence $\left(l_{n}\right)_{n=0}^{\infty} \in\{0,1\}^{\mathbb{N}}$. Put then

1. $q=0: l_{n} \equiv 0$;

2. $0<q<\aleph_{0}$ :

$$
l_{n}=\left\{\begin{array}{l}
1: 0 \leq n \leq q-1, \\
0: n \geq q ;
\end{array}\right.
$$

3. $r=0: l_{n} \equiv 1$;

4. $0<r<\aleph_{0}$ :

$$
l_{n}=\left\{\begin{array}{l}
0: 1 \leq n \leq r, \\
1: n=0 \text { or } n>r
\end{array}\right.
$$

5. $q=r=\aleph_{0}: l_{n}=\frac{1}{2}\left(1+(-1)^{n}\right)$.

We have defined the set $\mathcal{S}$. In order to show that the generated group $\mathcal{G}=\langle\mathcal{S}\rangle$ is an appropriate one we have to change the set of generators to obtain one with the properties $(\mathrm{G}),(\mathrm{P})$ and $(\mathrm{I})$.

Put

$$
F_{0}=S_{0}=J^{1-l_{0}} T^{l_{0}}, \quad F_{n}=T^{l_{0}} S_{k_{n}-1}^{-1} S_{k_{n+1}-1} T^{-l_{0}}, \quad n \geq 1
$$

and denote $\mathcal{F}=\left\{F_{n}: n \geq 0\right\}$. Since $\iota\left(k_{n}-1\right)=-n+1-l_{0}$ for $n \geq 1$, and $\iota\left(k_{n}\right)=k_{n}+l_{n}$ for $n \geq 0$,

$$
S_{k_{n}-1} S_{k_{n+1}-1}^{-1}=(J T)^{k_{n}} J(J T)^{-\left(k_{n}+l_{n}\right)}=S_{k_{n}}=S_{k_{n}+l_{n}}^{-1} .
$$


It follows that the set $\mathcal{F}$ generates $\mathcal{G}$ since $l_{n} \in\{0,1\}$. For $n \geq 1$ we have $F_{n}$ in the reducible form

$$
F_{n}= \begin{cases}(T J)^{-n+1} G_{l_{n}}(T J)^{n-1}: & l_{0}=1 ; \\ (J T)^{-n+1} T J T^{-1}(J T)^{n-1}: & l_{0}=0 .\end{cases}
$$

where $G_{j}=(J T)^{j+1} J T^{-1} J, j=0,1$, hence

$$
\left|F_{0}\right|=1, \quad\left|F_{n}\right|=\left|F_{n}^{-1}\right|=4 n-1+2\left(l_{n}+l_{0}\right), n \geq 1 .
$$

Recall that $l_{0}=0$ iff $q=0$ iff $l_{n} \equiv 0$.

By inspection one can check that $\mathcal{F}$ satisfies the property (I). Properties $(\mathrm{G})$ and $(\mathrm{P})$ of the set $\mathcal{F}$ for the case 1 . (and 2. with $q=1$ ) are shown in [6], hence assume that $l_{0}=1$ (i.e. we consider cases 2 . -5 .).

Observe that both $F_{n}$ and $F_{n}^{-1}, n \geq 1$, are of the irreducible form

$$
(T J)^{-n+1}\left[J T A T^{-1} J\right](T J)^{n-1} .
$$

By easy induction we get that an arbitrary product $F_{n_{1}}^{\varepsilon_{1}} \cdot \ldots \cdot F_{n_{t}}^{\varepsilon_{t}}$, where $\varepsilon_{j}= \pm 1$ and $n_{j} \neq 0, j=1, \ldots, t$, is of the irreducible form

$$
(T J)^{-n_{1}+1}\left[J T A T^{-1} J\right](T J)^{n_{t}-1},
$$

provided $n_{j}$ 's are pairwise distinct. This immediately implies the property (G). Moreover, it follows that if $A=(T J)^{-k+1}\left[J T B T^{-1} J\right](T J)^{k-1}$ and $C=$ $(T J)^{-l+1}\left[J T D T^{-1} J\right](T J)^{l-1}$, with arbitrary $B$ and $D$ such that the above forms are irreducible, then we have

$$
|A C|=|A|+|C|-4(k \wedge l)+1
$$

The above formula allows us to show the property $(\mathrm{P})$ of $\mathcal{F}$.

Indeed, let $G=F_{n_{1}}^{\varepsilon_{1}} \ldots F_{n_{t}}^{\varepsilon_{t}}, t \geq 2, \varepsilon_{j} \in\{-1,1\}, j=1,2, \ldots, t$. First observe that trivially we have

$$
|G|=\left|F_{n_{1}}^{\varepsilon_{1}} \ldots F_{n_{i-1}}^{\varepsilon_{i-1}}\right|+\left|F_{n_{i+1}}^{\varepsilon_{i+1}} \ldots F_{n_{t}}^{\varepsilon_{t}}\right|+1>1=\left|F_{n_{i}}^{\varepsilon_{i}}\right|
$$

if $n_{i}=0$. 
If we assume that $n_{j} \neq 0, j=1, \ldots, t$, then in virtue of (3.5) and (3.4) we have

$$
\begin{aligned}
|G|-\left|F_{n_{i}}\right|= & \sum_{j \neq i}\left|F_{n_{j}}^{\varepsilon_{j}}\right|+t-1-4 \sum_{j=1}^{t-1}\left(n_{j} \wedge n_{j+1}\right) \\
= & 4\left(\sum_{j \neq i} n_{j}-\sum_{j=1}^{t-1}\left(n_{j} \wedge n_{j+1}\right)\right)+2\left(t-1+\sum_{j \neq i} l_{n_{j}}\right) \\
= & 4\left(\sum_{j=1}^{i-1}\left(n_{j}-\left(n_{j} \wedge n_{j+1}\right)\right)+\sum_{j=i+1}^{t}\left(n_{j}-\left(n_{j-1} \wedge n_{j}\right)\right)\right) \\
& +2\left(t-1+\sum_{j \neq i} l_{n_{j}}\right) \\
\geq & 2\left(t-1+\sum_{j \neq i} l_{n_{j}}\right) \geq 2>0 .
\end{aligned}
$$

Now we deal with the case $p=2$.

To construct the appropriate $\iota$ put $k_{0}=0, l_{0}=9$ and then $\iota_{0}(0)=9$, $\iota_{0}(1)=4, \iota_{0}(2)=6, \iota_{0}(3)=7, \iota_{0}(5)=8$. For $n \geq 1$ define $\iota_{n}$ choosing $l_{n} \in\{0,1\}$. Next denote

$$
F_{n}= \begin{cases}S_{2} S_{1}^{-1} & : n=0 \\ S_{1} S_{2} S_{1}^{-1} & : n=1 \\ S_{k_{n-1}-1}^{-1} S_{k_{n}-1}: & n \geq 2 .\end{cases}
$$

Observe that $S_{1}^{-1} S_{2}=S_{3}, S_{2}^{-1} S_{3}=S_{5}, S_{1} S_{5}=S_{0}=S_{k_{1}-1}^{-1}$ and (3.2) holds for $n \geq 2$, hence by the analogous argument $\mathcal{F}=\left\{F_{n}: n \geq 0\right\}$ generates $\mathcal{G}$.

The irreducible form of $F_{n}$ is the following

$$
F_{n}= \begin{cases}J T J T J T^{-1} J T J & : n=0 ; \\ J T J T^{-1} J T J T J & n=1 ; \\ (J T)^{-n+2} H_{l_{n-1}}(J T)^{n-2}: & n \geq 2,\end{cases}
$$

where $H_{j}=T(J T)^{j} J T^{-1}, j=0,1$, hence

$$
\left|F_{0}\right|=\left|F_{1}\right|=9, \quad\left|F_{n}\right|=\left|F_{n}^{-1}\right|=4 n-5+2 l_{n-1}, n \geq 2 .
$$

Again by inspection one can check that $\mathcal{F}$ satisfies the property (I). 
Before we proceed to the proof of property $(\mathrm{P})$ denote $\mathbb{N}_{1}=\{0,1\}, \mathbb{N}_{2}=$ $\{2,3, \ldots\}, \mathcal{F}_{j}=\left\{F_{n}: n \in \mathbb{N}_{j}\right\}, j=1,2$, and note that

1. $\forall\left(\varepsilon_{k} \in\{-1,1\}, k=1,2 ; n \in \mathbb{N}_{i}, m \in \mathbb{N}_{j}, i, j \in\{1,2\}, i \neq j\right)$

$$
\left|F_{n}^{\varepsilon_{1}} F_{m}^{\varepsilon_{2}}\right|=\left|F_{n}\right|+\left|F_{m}\right|
$$

2. $\forall\left(\varepsilon_{k} \in\{-1,1\}, n_{k} \in \mathbb{N}_{1} \quad k=1,2 ;\right)$

$$
\left|F_{n_{1}}^{\varepsilon_{1}} F_{n_{2}}^{\varepsilon_{2}}\right|=\left\{\begin{array}{l}
\left|F_{n_{1}}\right|+\left|F_{n_{2}}\right|-3: \varepsilon_{1} \varepsilon_{2}=1 ; \\
\left|F_{n_{1}}\right|+\left|F_{n_{2}}\right|-7: \varepsilon_{1} \varepsilon_{2}=-1, n \neq m ;
\end{array}\right.
$$

3. $\forall\left(\varepsilon_{k} \in\{-1,1\}, n_{k} \in \mathbb{N}_{2} \quad k=1,2 ;\right)$

$$
\left|F_{n_{1}}^{\varepsilon_{1}} F_{n_{2}}^{\varepsilon_{2}}\right|=\left|F_{n_{1}}\right|+\left|F_{n_{2}}\right|-4\left(n_{1} \wedge n_{2}\right)+7 .
$$

In virtue of 1 . of (3.8) it is enough to show the property $(\mathrm{P})$ separately for the sets $\mathcal{F}_{1}$ and $\mathcal{F}_{2}$. Let $G=F_{n_{1}}^{\varepsilon_{1}} \cdot \ldots \cdot F_{n_{t}}^{\varepsilon_{t}}$. In the former case from 2. of (3.8) and the first part of (3.7) it follows that

$$
|G|-\left|F_{n_{i}}\right| \geq 9 t-7(t-1)-9=2(t-1) \geq 2>0 .
$$

In the latter case from 3. of (3.8) and the second part of (3.7) it follows that

$$
\begin{aligned}
|G|-\left|F_{n_{i}}\right| & =\sum_{j \neq i}\left|F_{n_{j}}^{\varepsilon_{j}}\right|-4 \sum_{j=1}^{t-1}\left(n_{j} \wedge n_{j+1}\right)+7(t-1) \\
& =4\left(\sum_{j \neq i} n_{j}-\sum_{j=1}^{t-1}\left(n_{j} \wedge n_{j+1}\right)\right)+2\left(t-1+\sum_{j \neq i} l_{n_{j}-1}\right) \\
& \geq 2\left(t-1+\sum_{j \neq i} l_{n_{j}-1}\right) \geq 2>0 .
\end{aligned}
$$

Considerations similar to those in the previous case convince us that the property $(\mathrm{G})$ is satisfied. Therefore Lemma 1.1 tells us that $\mathcal{F}=\mathcal{K}(\mathcal{G})$ is satisfied in each considered case. Now from Hall's proof of the Kurosh subgroup theorem it follows that $\mathcal{G} \simeq \mathcal{F}_{p} * \mathcal{T}_{q} * \mathcal{D}_{r}$ since, in virtue of (3.3), (3.6) and the definition of $\left(l_{n}\right)$, the set $\mathcal{F}$ consists of an appropriate number of elements of infinite order, elements of order three and involutions.

From (3.3) and (3.6) it immediately follows that (2.3) is satisfied in all cases. In virtue of Lemma 2.1 each of $(\mathcal{G}, \mathcal{S})$ is a Cayley representation of the distant graph on $\mathbb{P}(\mathbb{Z})$.

We are not able to find a building involution that produces an odd number of independent generators of infinite order. Taking this into account and based on the above proof, we make the following conjecture. 
Conjecture 3.2. A subgroup of $\operatorname{PSL}(2, \mathbb{Z})$ is a group of Cayley representation of the distant graph on $\mathbb{P}(\mathbb{Z})$ if and only if it is of the form (3.1), where $p \in$ $2 \mathbb{N} \cup\left\{\aleph_{0}\right\}, q, r \in \mathbb{N} \cup\left\{\aleph_{0}\right\}$ and $p+q+r=\aleph_{0}$.

Open Access. This article is distributed under the terms of the Creative Commons Attribution 4.0 International License (http://creativecommons.org/licenses/ by/4.0/), which permits unrestricted use, distribution, and reproduction in any medium, provided you give appropriate credit to the original author(s) and the source, provide a link to the Creative Commons license, and indicate if changes were made.

\section{References}

[1] Blunck, A., Havlicek, H.: On distant-isomorphisms of projective lines. Aequ. Math. 69, 146-163 (2005)

[2] Blunck, A., Havlicek, H.: Radical parallelism on projective lines and non-linear models of affine spaces. Math. Pannon. 14, 113-127 (2003)

[3] Blunck, A., Herzer, A.: Kettengeometrien-Eine Einfuhrung. Shaker Verlag, Aachen (2005)

[4] Hall Jr., M.: Subgroups of free produts. Pac. J. Math. 3, 115-120 (1953)

[5] Matraś, A., Siemaszko, A.: The shortest path problem for the distant graph of the projective line over the ring of integers. Bull. Malays. Math. Sci. Soc. 41, 231-248 (2018)

[6] Matraś, A., Siemaszko, A.: The Cayley property of some distant draphs and relationship with the Stern-Brocot tree. Results Math. 73, 14 (2018)

Andrzej Matraś and Artur Siemaszko

Faculty of Mathematics and Computer Science

University of Warmia and Mazury in Olsztyn

Słoneczna 54

10-790 Olsztyn

Poland

e-mail: artur@uwm.edu.pl

Andrzej Matraś

e-mail: matras@uwm.edu.pl

Received: January 9, 2019.

Accepted: March 9, 2019.

Publisher's Note Springer Nature remains neutral with regard to jurisdictional claims in published maps and institutional affiliations. 